\title{
Stochastic price based coordinated operation planning of energy storage system and conventional power plant
}

\author{
Mohammad Reza SHEIBANI ${ }^{1}$, Gholam Reza YOUSEFI ${ }^{1}$ (D, \\ Mohammad Amin LATIFY ${ }^{1}$
}

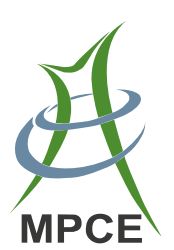

\begin{abstract}
A generation company (GENCO) which has a conventional power plant (CPP) intends to add an energy storage system (ESS) beside the CPP to increase its flexibility and profitability. For this purpose, a new model is proposed for coordinated operation planning of the CPP and ESS in energy and spinning reserve markets in the presence of a bilateral contract. The proposed model is based on the stochastic price based unit commitment (PBUC) and price based storage commitment (PBSC). The uncertainties of the energy and spinning reserve prices and delivery requests in the spinning reserve market are modeled via scenarios based upon historical data. The proposed model maximizes the profitability of the ESS beside the CPP and encourages the GENCO to invest the ESS. ESS technology options to use beside the CPP are determined by economic assessments. Numerical results show that utilization of ESSs improves the technical operation of CPPs, as well as GENCOs' profitability.
\end{abstract}

Keywords Energy storage system, Conventional power plant, Operation planning, Price based unit commitment

CrossCheck date: 8 March 2019

Received: 27 February 2018/Accepted: 8 March 2019/Published online: 15 July 2019

(C) The Author(s) 2019

$\triangle$ Gholam Reza YOUSEFI

yousefi@cc.iut.ac.ir

Mohammad Reza SHEIBANI

m.sheibani@ec.iut.ac.ir

Mohammad Amin LATIFY

latify@cc.iut.ac.ir

1 Department of Electrical and Computer Engineering, Isfahan University of Technology, Isfahan 84156-83111, Iran

\section{Introduction}

\subsection{Motivation}

In recent years, researchers have been sought energy management strategies allowing a more flexible and controllable operation [1]. Recent development and advance in energy storage system (ESS) technologies have made the application of these systems a viable solution to improve the flexibility of power systems [2-6].

Conventional power plants (CPPs), and especially the thermal generators, due to their technical limitations such as minimum generation capability, ramp rate, and minimum up and down time restrictions, have limited flexibility. These limitations reduce the cycling capability of power plants. Cycling is changing the power output of CPPs by means of ramping and switching (starting up and shutting down) [7]. Cycling capability of CPPs is an important feature to participate in ancillary service markets. The limitations of CPPs may also reduce their profitability, especially in the presence of bilateral contracts.

Installing ESSs beside CPPs could mitigate the foregoing problems. The flexible charging/discharging characteristics of the ESSs can improve the controllability of the CPPs. In this paper, we propose a new model for coordinated economic operation of an ESS with a CPP in a dayahead market in the presence of a bilateral contract.

\subsection{Literature review}

Recently, a large number of researches have been carried out on the optimal operation of ESSs beside renewable energy sources (RESs) from GENCOs' point of view [8-11]. ESSs can alleviate the problems caused by the uncertain and variable generation of RESs. 
Only a few works have studied the joint operation of ESSs with CPPs in GENCOs. Some studies have focused on integrating ESSs with RESs and CPPs in virtual power plants. In [12], a weekly self-scheduling of a virtual power plant in a day-ahead energy market and a bilateral contract are presented. A two-stage stochastic offering model for a virtual power plant in the day-ahead and balancing markets is addressed [13]. A probabilistic model for optimal dayahead scheduling of a virtual power plant in a day-ahead electricity market is proposed in [14, 15]. In [16], a probabilistic model for optimal day-ahead scheduling of a virtual power plant in energy and reserve markets is proposed. A day-ahead scheduling framework for a virtual power plant in a joint energy and regulation reserve markets is present in [17].

In the aforementioned papers, the virtual power plant sells and purchases electricity in a day-ahead market seeking to maximize its expected profit. In these papers, ESSs are used to settle the uncertainty of RESs. The ESSs can be charged using the RESs and purchasing power from the market. The impacts of the ESSs on technical limitations of the CPPs are not studied in these papers. Also, the opportunities of power exchanges between the ESSs, CPPs, and day-ahead markets to increase the GENCOs' profits are not modeled. According to the operation strategies suggested in the aforementioned research works, in the absence of RESs, ESSs are not economically profitable.

In the literature the only situation in which storage systems are used beside the CPPs (in the absence of RESs) is the combination of the thermal storage systems and combined heat and power (CHP) plants [18, 19]. The CHP plants simultaneously produce heat and electrical power. In [20], the optimal integration of a heat storage system into a heating network is studied. The purpose of this study is to minimize the cost of heat. The CHP is profitable when a sufficient heat demand is requested or when the CHP is connected to a heat network. The optimal day-ahead scheduling of ESSs and thermal energy storage systems beside the CHPs is addressed in [21]. In this work the ESS is independently linked to the power grid and energy exchanges between the ESS and the CHPs are not considered. In [22, 23], the thermal storage systems are used to increase the flexibility of the CHPs.

In the aforementioned papers, only thermal ESSs connected to CHPs are studied. The proposed strategies in these studies are not suitable for the other CPP and ESS technologies.

\subsection{Contribution}

The purpose of this paper is to demonstrate the ability of ESSs to improve the performance of a CPP when participating in a day-ahead market and providing bilateral contracts. In the literature the impacts of ESSs on the performance of the CPPs are not modeled. In these studies the ESSs are used to support RESs uncertainties. Therefore, according to the proposed operation strategies in these papers, the ESSs are economic only if they are utilized beside the RESs, and in the absence of RESs they are not economically profitable.

In the proposed model in present manuscript, the capability of power exchanges between an ESS, a CPP and the electricity market, cycling cost of the CPP, and impact of the ESS on providing bilateral contracts are considered. Indeed, a new model is proposed for the coordinated operation planning of the ESS and CPP problem aimed to participate in the day-ahead market and provide bilateral contracts. The results of studies show that using the proposed operation strategy, the utilization of ESSs beside the CPPs is economically justifiable. Also, ESSs are able to increase the flexibility and efficiency of CPPs. This increase is useful from two aspects, explained as follows.

1) In the presence of bilateral contracts, the CPP sometimes must generate power lower than rated. This problem reduces the efficiency of the CPP. The ESS can help the CPP to overcome this drawback.

2) In the future with increasing penetration of RESs, CPPs must be more flexible. This problem leads to the increase of operation and maintenance $(\mathrm{O} \& \mathrm{M})$ costs of CPPs and the decrease of their efficiency and life time [24]. In this situation the ESSs are able to improve the performance of the CPPs.

In this work, a new stochastic strategy for coordinated operation planning of the CPP and ESS is proposed. The presented method maximizes the profitability of the ESS beside the CPP. This method is based on a coordinated price based unit commitment (PBUC) and price based storage commitment (PBSC) in energy and ancillary services markets in the presence of a bilateral contract. The uncertainties of the energy and spinning reserve prices and spinning reserve delivery requests are modeled based on a scenario-based stochastic programming.

Thus, the main contributions and novelties of this paper are as follows:

1) Modeling the coordinated committing of a CPP and an ESS in a day-ahead market and a bilateral contract.

2) Employing the ESS to improve the flexibility and profitability of the GENCO and improve the technical operation of the CPP.

3) Proposing number of payback cycles as a new economic criterion to assess ESS technologies. 


\subsection{Paper organization}

Section 2 presents the model proposed for coordination operation planning of the CPP and the ESS. Module formulation and scenario generation and reduction description are provided in this section as well. Numerical studies and economic assessments are presented in Section 3. Finally, Section 4 provides the concluding remarks.

\section{Model description}

In this paper, it is assumed that a price taker GENCO is the owner of a CPP. The GENCO wants to use an ESS beside the CPP to improve the economic and technical operation of the CPP. Herein, a new model is presented for the joint operation planning of the CPP and ESS. In this model, PBUC and PBSC are determined for the GENO in the day-ahead energy and spinning reserve markets. PBUC determines the generation unit's ON/OFF status, based on the market prices and technical characteristics of the units, in order to maximize the GENCO's profit. This paper introduces the PBSC as a new concept. The PBSC determines the charge, discharge, and idle modes of the ESS based on the market prices and the ESS technical characteristics. In the idle mode, the ESS does not charge or discharge.

In the proposed model, the coordinated PBUC and PBSC problem is solved as a single problem by a GENCO. The simultaneous PBUC/PBSC practically provides the option of the power exchange between the CPP and the

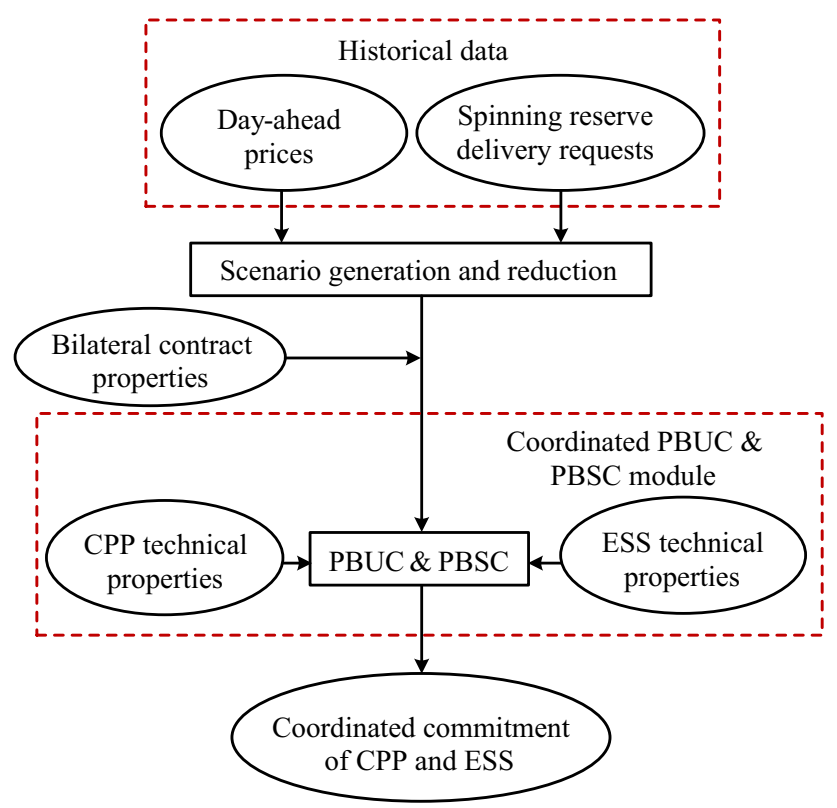

Fig. 1 Coordinated PBUC and PBSC module
ESS. Figure 1 depicts the model proposed for the coordinated PBUC and PBSC. The energy and spinning prices and spinning reserve delivery requests are probabilistically modeled and are the inputs of the simultaneous PBUC and PBSC module. The technical properties of the CPP and the ESS are the inputs of this module as well. To show the ability of the proposed model to increase the flexibility of the CPP, in particular in the presence of GENCO's obligations, a bilateral contract is considered as the generation obligation. The power and the price of the bilateral contract are the other inputs of the module. The output of this module is the simultaneous commitment of the CPP and the ESS in the day-ahead energy and ancillary services markets and the bilateral contract.

The formulation of the PBUC and the PBSC is explained in the following. Then, the scenario generation and reduction of the uncertainty parameters are discussed.

\subsection{Coordinated PBUC \& PBSC module formulation}

The power exchange scheme considered in the simultaneous PBUC and PBSC module is depicted in Fig. 2. The power exchange opportunities between the ESS, CPP, dayahead market, and bilateral contract are shown in this figure. The CPP can participate in the day-ahead energy and spinning reserve markets. The CPP can also charge the ESS. This is done when the CPP is ON and not completely dispatched in the market and the bilateral contract. The power flow direction between CPP and ESS is unidirectional. The energy stored in the ESS can be used at other time for the price arbitrage. The ESS can also be charged by receiving power from the day-ahead energy market. The ESS can be discharged to participate in the day-ahead energy and spinning reserve markets and bilateral contract. The power flow direction between day-ahead market and ESS is bi-directional.

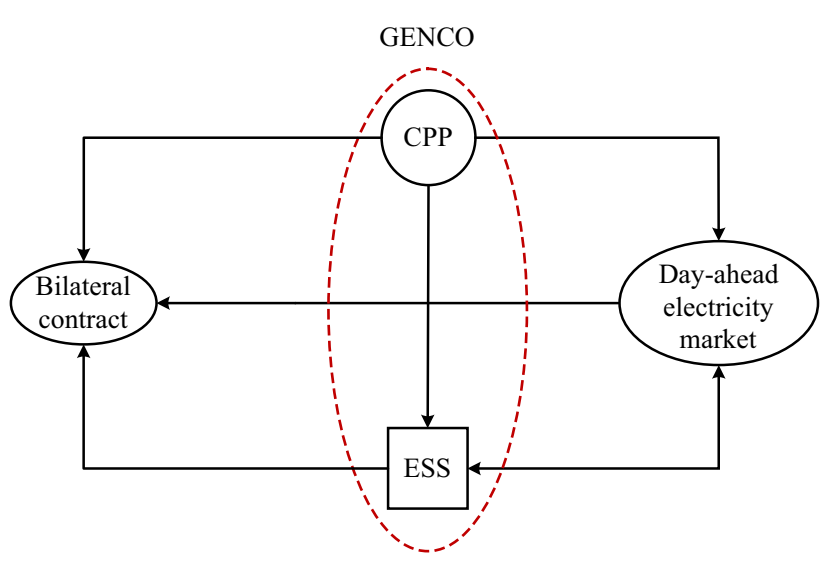

Fig. 2 Power exchange scheme in the proposed model 
The GENCO can provide the bilateral contract power by the CPP, ESS, or purchasing power from the day-ahead energy market. In the proposed model, the presence of the ESS improves the CPP operation and economic benefits in different ways as follows.

1) The combination of the ESS and the CPP increases the price arbitrage opportunities for the GENCO. The ESS can be charged by the CPP or by purchasing power from the day-ahead market, and can be discharged for committing in the day-ahead market or for providing a portion of the bilateral contract.

2) The presence of the ESS helps to increase the GENCO's flexibility, which is limited by the ramp rate constraints of the CPP, for providing the ancillary services. Provision of the ancillary services by the CPP leads to cycling problems. Cycling refers to the operation of electric generation units at varying load levels, including ON/OFF, load following, and minimum load operation, in response to changes in the system load requirements. This increases the maintenance costs and forced outages of the CPP [7].

The objective function of the simultaneous PBUC and PBSC module maximizes the expected profit of the GENCO, as shown in (1).
0 means spinning reserve delivery is not requested); $R_{s}^{c}(t)$ is spinning reserve offer of the CPP at time $t$ and scenario $s$; $R_{s}^{s, c h}(t)$ and $R_{s}^{s, d i s}(t)$ are the spinning reserve offer of the ESS in charging and discharging modes at time $t$ and scenario $s$, respectively; $P^{b}(t)$ and $\rho^{b}(t)$ are the bilateral contract power and price at time $t$, respectively; $M C^{c}$ is the marginal cost of the CPP at minimum generating capacity; $I(t)$ is the status indicator for the CPP ( 1 means $\mathrm{ON}$ and 0 means OFF); $N$ is the number of segments of the piecewise linearized cost curve; $\mu^{n}$ is the slope of the $n^{\text {th }}$ segment in the linearized cost curve; $p_{s}^{n}(t)$ is the production in the $n^{\text {th }}$ segment at time $t$ and scenario $s ; S U(t)$ and $S D(t)$ are the startup and shutdown costs of the CPP at time $t$; and $C_{s}^{c, \text { ramp }}(t)$ is the ramping cost of the CPP at time $t$ and scenario $s$.

The first term in (1) models the power exchange between the GENCO and the energy market. The second term represents the revenues of the CPP and the ESS in the day-ahead spinning reserve market. If the spinning reserve delivery is requested, the GENCO receives both energy and spinning reserve prices; but when spinning reserve delivery is not requested, the GENCO receives only the spinning reserve price.

The third term in (1) determines the revenue of the bilateral contract. The forth term represents the cost of the

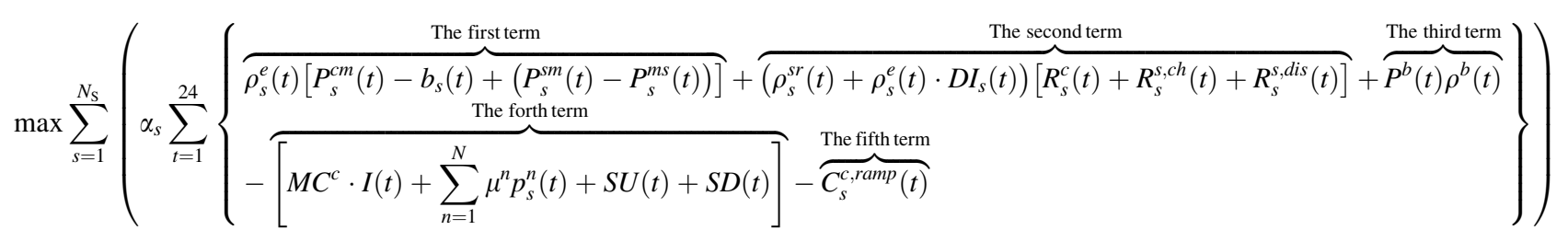

where $s$ is the scenario index; $t$ is the hour index; $n$ is the segment index in linearized cost curve; $N_{S}$ is the number of scenarios; $\alpha_{s}$ is the probability of scenario $s ; \rho_{s}^{e}(t)$ and $\rho_{s}^{s r}(t)$ are the energy price and spinning reserve price at time $t$ and scenario $s$, respectively; $P_{s}^{c m}(t)$ is the generation of the CPP in the energy market at time $t$ and scenario $s ; b_{s}(t)$ is the power purchased from the energy market for the bilateral contract at time $t$ and scenario $s ; P_{s}^{s m}(t)$ is the amount of power delivered while discharging the ESS for the energy market at time $t$ and scenario $s ; P_{s}^{m s}(t)$ is the amount of charging power of the ESS by purchasing power from the energy market at time $t$ and scenario $s ; D I_{s}(t)$ is the spinning reserve delivery request indicator at time $t$ and scenario $s$ ( 1 means spinning reserve delivery is requested and power provided by the CPP. In this term, the cost function of the CPP is represented by the piece-wise linear function. The fifth term represents the ramping cost of the CPP.

The output power of the CPP is the sum of the powers that are generated for the day-ahead energy market, bilateral contract, and $P$ charging of the ESS, as follows:

$P_{s}^{c}(t)=P_{s}^{c m}(t)+P_{s}^{c b}(t)+P_{s}^{c s}(t)$

where $P_{s}^{c}(t)$ is the generation of the CPP at time $t$ and scenario $s ; P_{s}^{c b}(t)$ and $P_{s}^{c s}(t)$ are the generation of the CPP for the bilateral contract and for charging of the ESS at time $t$ and scenario $s$, respectively.

The cost function of the CPP is represented by a piecewise linear function as follows: 
$P_{s}^{c}(t)+R_{s}^{c}(t) \cdot D I_{s}(t)=\underline{P}^{c} I(t)+\sum_{n=1}^{N} p_{s}^{n}(t) \quad \forall t, \forall s$

$0 \leq p_{s}^{n}(t) \leq \bar{p}^{n} I(t) \quad \forall t, \forall s, \forall n$

where $\underline{P}^{c}$ is the minimum capacity of the CPP; and $\bar{p}^{n}$ is the maximum generation capacity of the CPP in the $n^{\text {th }}$ segment. Equations (5)-(14) represent the CPP constraints.

$P_{s}^{c}(t)-P_{s}^{c}(t-1) \leq R U^{c} \cdot I(t) \quad \forall t, \forall s$

$P_{s}^{c}(t-1)-P_{s}^{c}(t) \leq R D^{c} \cdot I(t-1) \quad \forall t, \forall s$

$\sum_{t^{\prime}=t}^{t+T^{o n}-1} I\left(t^{\prime}\right) \geq T^{o n}(I(t)-I(t-1)) \quad \forall t \in\left\{1,2, \ldots, 25-T^{o n}\right\}$

$\sum_{t^{\prime}=t}^{t+T^{o f f}-1}\left(1-I\left(t^{\prime}\right)\right) \geq T^{o f f}(I(t-1)-I(t))$

$\forall t \in\left\{1,2, \ldots, 25-T^{o f f}\right\}$

$S U(t) \geq K^{c}(I(t)-I(t-1)) \quad \forall t$

$S D(t) \geq J^{c}(I(t-1)-I(t)) \quad \forall t$

$0 \leq R_{s}^{c}(t) \leq 10 \cdot \operatorname{MSR}^{c} \quad \forall t, \forall s$

$P_{s}^{c}(t)+R_{s}^{c}(t) \leq \bar{P}^{c} I(t) \quad \forall t, \forall s$

$\underline{P}^{c} I(t) \leq P_{s}^{c}(t) \leq \bar{P}^{c} I(t) \quad \forall t, \forall s$

$0 \leq P_{s}^{c b}(t) \leq P^{b}(t) I(t) \quad \forall t, \forall s$

where $R U^{c}$ and $R D^{c}$ are the ramp up and down of the CPP, respectively; $T^{o n}$ and $T^{\text {off }}$ are the minimum uptime and off time of the CPP, respectively; $K^{c}$ and $J^{c}$ are the startup and shutdown costs of the CPP, respectively; $M S R^{c}$ is the maximum sustained ramp rate of the CPP; and $\underline{P}^{c}$ is the minimum generation capacity of the CPP. In (5)-(8), ramping up and down limitations and minimum up and down times constraints of the CPP are shown, respectively. In (9) and (10), startup and shutdown costs of the CPP are determined, respectively. 10-minute spinning reserve constraint of the CPP is applied by (11). Maximum and minimum generation limitations of the CPP are shown in (12) and (13). As shown in (14), the power generated by the CPP for the bilateral contract should be lower than the bilateral contract power. This power is provided when the CPP is ON.

The power purchased from the day-ahead energy market for the bilateral contract should be lower than the bilateral contract power as follows:

$0 \leq b_{s}(t) \leq P^{b}(t) \quad \forall t, \forall s$

Ramping cost of the CPP are determined as follows:

$$
\begin{aligned}
& C_{s}^{c, \operatorname{ramp}}(t) \geq R C^{c} \cdot\left(P_{s}^{c}(t)-P_{s}^{c}(t-1)+R_{s}^{c}(t) \cdot D I_{s}(t)\right) \\
& \quad \forall t, \forall s \\
& C_{s}^{c, r a m p}(t) \geq R C^{c} \cdot\left(P_{s}^{c}(t-1)-P_{s}^{c}(t)+R_{s}^{c}(t) \cdot D I_{s}(t)\right) \\
& \quad \forall t, \forall s
\end{aligned}
$$

$C_{s}^{c, \operatorname{ramp}}(t) \geq R C^{c} \cdot R_{s}^{c}(t) \cdot D I_{s}(t) \quad \forall t, \forall s$

where $R C^{c}$ is the ramping cost of the CPP. Equations (19)(29) represent the ESS constraints.

$$
\begin{gathered}
\sum_{t=1}^{T} \eta_{c h}\left(P_{s}^{m s}(t)+P_{s}^{c s}(t)-D I_{s}(t) \cdot R_{s}^{s, c h}(t)\right)=\sum_{t=1}^{T} \frac{1}{\eta_{d i s}} . \\
\left(P_{s}^{s m}(t)+P_{s}^{s b}(t)+D I_{s}(t) \cdot R_{s}^{s, d i s}(t)\right) \quad \forall t, \forall s \\
E_{s}(t)=E_{s}(t-1)+\eta_{c h}\left(P_{s}^{m s}(t)+P_{s}^{c s}(t)-D I_{s}(t) \cdot R_{s}^{s, c h}(t)\right) \\
-\frac{1}{\eta_{d i s}}\left(P_{s}^{s m}(t)+P_{s}^{s b}(t)+D I_{s}(t) \cdot R_{s}^{s, d i s}(t)\right) \quad \forall t, \forall s
\end{gathered}
$$

$\underline{E}^{s} \leq E_{s}(t) \leq \bar{E}^{s} \quad \forall t, \forall s$

$0 \leq P_{s}^{m s}(t), P_{s}^{s m}(t), R_{s}^{s, c h}(t), R_{s}^{s, d i s}(t), P_{s}^{c s}(t), P_{s}^{s b}(t) \leq \bar{P}^{s}$

$\forall t, \forall s$

$0 \leq P_{s}^{m s}(t)+P_{s}^{c s}(t) \leq \bar{P}^{s} \cdot \operatorname{ch}(t) \quad \forall t, \forall s$

$0 \leq P_{s}^{s m}(t)+R_{s}^{s, d i s}(t)+P_{s}^{s b}(t) \leq \bar{P}^{s} \cdot \operatorname{dis}(t) \quad \forall t, \forall s$

$R_{s}^{s, d i s}(t) \leq \eta_{\text {dis }} E_{s}(t-1)-\left(P_{s}^{s m}(t)+P_{s}^{s b}(t)\right) \quad \forall t, \forall s$

$\operatorname{ch}(t)+\operatorname{dis}(t) \leq 1 \quad \forall t$

$0 \leq R_{s}^{s, d i s}(t) \leq 10 \cdot M_{S R}^{s} \quad \forall t, \forall s$

$0 \leq R_{s}^{s, c h}(t) \leq P_{s}^{m s}(t)+P_{s}^{c s}(t) \quad \forall t, \forall s$

$0 \leq P_{s}^{s b}(t) \leq P^{b}(t) \quad \forall t, \forall s$

where $\eta_{c h}$ and $\eta_{\text {dis }}$ are the charging and discharging efficiencies of the ESS, respectively; $P_{s}^{s b}(t)$ is the generation of the ESS for the bilateral contract at time $t$ and scenario $s$; $E_{s}(t)$ is the energy of the ESS at time $t$ and scenario $s ; \underline{E}^{s}$ and $\bar{E}^{s}$ are the minimum and maximum energy capacities of the ESS, respectively; $\bar{P}^{s}$ is the maximum power capacity of the ESS; $c h(t)$ is the ESS charging status indicator at time $t$ ( 1 means charging and 0 means not charging); dis $(t)$ is the ESS discharging status indicator at time $t$ ( 1 means discharging and 0 means not discharging); and $M S R^{s}$ is the maximum sustained ramp rate of the ESS. Power balance equation of the ESS is shown in (19). The 
ESS can participate in the spinning reserve market in the charging and discharging modes. In the charging mode, the ESS can readily reduce its charging power and consequently reduce the overall system load. State of charge of the ESS is defined by (20). The energy and power capacities constraints of the ESS are applied by (21)-(24). According to (25), the ESS does not offer to the spinning reserve market more than the stored energy in the ESS. Using (26), the ESS would not be allowed to be charged and discharged at the same time. The spinning reserve constraint of the ESS in the discharging and charging modes are shown in (27) and (28), respectively. According to (29), the discharging power for the bilateral contract should be lower than the bilateral contract power. The bilateral contract power is provided by the CPP, ESS and purchasing power from the energy market as follows:

$P^{b}(t)=P_{s}^{c b}(t)+P_{s}^{s b}(t)+b_{s}(t) \quad \forall t, \forall s$

The ESS can be charged by the CPP to the maximum generation capacity of the CPP when the CPP is $\mathrm{ON}$ as follows:

$0 \leq P_{s}^{c s}(t) \leq \bar{P}^{c} I(t) \quad \forall t, \forall s$

It is supposed that the amount of stored energy in the ESS in the last hour of a day and last hour of the previous day must be equal as follows:

$E_{s}(0)=E_{s}(T)=\underline{E}^{s} \quad \forall s$

\subsection{Scenario generation and reduction}

In this paper, the uncertainties of the energy and spinning reserve prices and spinning reserve delivery request at each hour are modeled based on a scenario generation and reduction method. In this method, to generate scenarios for the energy and spinning reserve prices, time series of these prices are generated based on the historical prices.

The autoregressive integrated moving average (ARIM) model is a linear model that is used to represent the time series of the prices [25]. To generate the price scenarios, at first, the degrees of the ARIMA model are determined based on the autocorrelation function (ACF) and partial autocorrelation function (PACF) of the time series observations. Then the ARIMA coefficients with respect to the selected ARIMA model are estimated by the maximum likelihood method. After the determination of the ARIMA model and its coefficients, the price scenarios are generated.

The spinning reserve delivery request is a binary parameter. It is equal to 1 if the spinning reserve delivery is requested; otherwise, it is equal to 0 . The procedure which is used to generate a scenario for spinning reserve delivery requests for all hours of a day is described as follows.
Probability mass function of the spinning reserve delivery request at each hour is defined as follows:

$P\left(D I_{s}(t)=1\right)=\zeta(t) \quad \forall t$

$P\left(D I_{s}(t)=0\right)=1-\zeta(t) \quad \forall t$

where $\zeta(t)$ is the probability of the spinning reserve delivery request at hour $t$. $\zeta(t)$ is calculated using the historical data. The interval $[0,1]$ is divided into subintervals as follows:

$A_{0}(t)=[0, \zeta(t)] \quad \forall t$

$A_{1}(t)=[\zeta(t), 1] \quad \forall t$

A string of 24 random numbers between 0 and 1 is generated by a random number generator with uniformly distribution. If the generated number at hour $t$ belongs to $A_{0}(t), D I_{s}(t)=1$; and if this number belongs to $A_{l}(t)$, $D I_{s}(t)=0$. The explained method can be repeated to generate different spinning reserve delivery request scenarios.

The large number of scenarios makes the problem intractable. Therefore, a suitable technique is required to reduce the number of scenarios. In this work, the fast forward selection algorithm is used for scenario reduction. This algorithm is based on the Kantorovich distance which is a probability distance [26]. The iterative fast forward selection algorithm starts without any scenario. In each iteration, the scenario that minimizes Kantorovich distance between selected and initial set is selected. It ends when a specified number of selected scenarios is reached. Then the probability of each non-selected scenario is transferred to its closest selected scenario.

\section{Numerical results}

To illustrate the capability of the proposed model, we consider a price taker GENCO which has installed an ESS beside a CPP and participates in the day-ahead energy and spinning reserve markets. The GENCO has a bilateral contract as well. The New York independent system operation (NY ISO) day-ahead market of is used as the case study. To show the effectiveness and robustness of the proposed approach, a comprehensive study is performed on three case studies (G1, G2 and G3). The operation characteristics of G1, G2 and G3 are provided in Table 1 [27]. G1, G2, G3 are studied as typical technologies for the intermediate load, base load, and peak load power plants, respectively. A $10 \mathrm{MW} / 20 \mathrm{MWh}$ (i.e. an ESS that has 10 MW capacity and capability of 20 MWh energy storage) ESS with $85 \%$ round trip efficiency and complete charging and discharging capability is assumed to be connected to 
Table 1 Operating characteristics of CPPs

\begin{tabular}{|c|c|c|c|c|c|c|c|c|c|c|c|c|}
\hline CPP & $a(\$)$ & $\begin{array}{l}b(\$ / \\
\text { MW) }\end{array}$ & $\begin{array}{l}c(\$ / \\
\left.\mathrm{MW}^{2}\right)\end{array}$ & $\begin{array}{l}K^{c} \\
(\$)\end{array}$ & $\begin{array}{l}J^{c} \\
(\$)\end{array}$ & $\frac{P^{c}}{(\mathrm{MW})}$ & $\begin{array}{l}\bar{P}^{c} \\
(\mathrm{MW})\end{array}$ & $\begin{array}{l}T^{o n} \\
\text { (hour) }\end{array}$ & $\begin{array}{l}T^{\text {off }} \\
\text { (hour) }\end{array}$ & $\begin{array}{l}M S R^{c}(\mathrm{MW} / \\
\mathrm{min})\end{array}$ & $\begin{array}{l}R C^{c}(\$ / \\
\mathrm{MW})\end{array}$ & $\begin{array}{l}R U^{c} \text { and } R D^{c} \\
(\mathrm{MW} / \mathrm{h})\end{array}$ \\
\hline G1 & 130 & 35 & 0.001 & 200 & 100 & 10 & 100 & 3 & 2 & 0.8 & 1 & 50 \\
\hline G2 & 50.4 & 35.4 & 0.025 & 50 & 50 & 15 & 75 & 4 & 4 & 2 & 0.7 & 60 \\
\hline G3 & 138 & 45 & 0.01 & 30 & 30 & 8 & 30 & 1 & 1 & 0.6 & 0.8 & 45 \\
\hline
\end{tabular}

the CPP. It is assumed that the ESS charging and discharging efficiencies are both equal to the square root of the round-trip efficiency and ESS does not have any ramp rate constraint. The day-ahead energy and spinning reserve prices of the New York Independent System Operator (NY ISO) market in August 2015 are used for the scenario generation [28]. The averages of the day-ahead prices in August 2015 for each hour of the day are used as the historical sample of prices. The seasonal variations of the sample are removed using the difference process. Then the candidate time series model of the historical sample is determined. The degree of the candidate time series model is determined based on the ACF and PACF of the time series samples using MATLAB software. Then the ARIMA coefficients are estimated. In the next step the obtained ARIMA model is verified. The ARIMA model has a zeromean white noise process. 100 price scenarios are generated using the proposed ARIMA model and MATLAB software. 100 spinning reserve delivery request scenarios are also generated using the generation method explained in Section 2.2. The probability of the spinning reserve delivery request is considered 0 during 1:00 to 6:00 and 0.03 during 7:00 to 24:00, respectively. Thus, a scenario tree with 10000 scenarios is developed with combination of the prices and spinning reserve delivery request scenarios. The generated scenarios are then reduced to 10 scenarios using scenario reduction technique explained in Section 2.2. Indeed, the iterative fast forward selection algorithm ends when 10 scenarios are reached. The simulation time before the scenario reduction is about the 4 hours, but after the scenario reduction this time is reduced to 20 seconds. The difference between the generation commitment results before and after the scenarios reduction is about the $4 \%$. Then, the scenarios reduction is reasonable.

In this paper, the coordinated commitment of the CPP and the ESS, explained in Section 2, is modeled as a mixed integer linear programming and solved by the MIP solver in GAMS 21.7 on a personal computer with 4 GB RAM and $2.68 \mathrm{GHz}$ processor.

The results of the coordinated PBUC (G1) and PBSC, with a $30 \mathrm{MW}$ bilateral contract at the price of $45 \$ / \mathrm{MWh}$ for a scenario with high probability are depicted in Table 2.
At first, the forecasted energy and spinning reserve prices and spinning reserve delivery requests are shown. In the fifth column, the CPP commitment status (CCS) is presented. In this column, 0 means that the unit is uncommitted while 1 indicates that the unit is committed. In the sixth column, the ESS commitment status (ECS) is presented. Therein, $1,-1$, and 0 show the charging, discharging, and idle modes of the ESS, respectively. At hours of the day when the market price is relatively low $(2: 00$, 3:00 and 4:00), the ESS is charged by purchasing power from the market. By increasing the price at 6:00, the ESS provides a portion of the bilateral contract. Without the presence of the ESS, at this hour the CPP has to be ON and generate the minimum generation capacity or purchase power from the market. At 7:00, the CPP is started. At this time, the GENCO, in addition to generate power for the day-ahead energy market, generates power to charge the ESS. At 13:00 the spinning reserve is called. The ESS is charged at 14:00 and 15:00 by the CPP. At 17:00 and 18:00, the ESS is discharged for the day-ahead energy market, because the price of energy is relatively high. At 21:00 and 22:00, the CPP charges the ESS to provide a portion of the bilateral contract at 23:00. At this hour, in the absence of the ESS, the GENCO has to hold the CPP $\mathrm{ON}$ or purchase power from the market for the bilateral contract, which may lead to the reduction of GENCO's profit.

The results show that the presence of the ESS beside the CPP improves the operation of the CPP, in addition to the GENCO's profit increase. To illustrate the impacts of using the ESS beside G1, the operation of the GENCO with and without the ESS in a scenario with high probability, are compared in Figs. 3, 4, 5, 6. For a 15 MW bilateral contract, the GENCO's profit, with and without the ESS, with respect to the bilateral contract price are depicted in Fig. 3. As the bilateral contract price increases, the profit of the GENCO increases as well. Furthermore, the GENCO's profit in the case of combined CPP and ESS is higher than the single CPP. For the mentioned bilateral contract in the cases of the single CPP and combined CPP and ESS, the number of hours when the CPP is ON with respect to the bilateral contract price, is shown in Fig. 4. For $15 \mathrm{MW}$ bilateral contract, the committed hours of the CPP has not 
Table 2 Coordinated PBUC and PBSC results (G1 + ESS)

\begin{tabular}{|c|c|c|c|c|c|c|c|c|c|c|}
\hline Time & $\begin{array}{l}\text { Forecasted } \\
\rho^{e}(t)\end{array}$ & $\begin{array}{l}\text { Forecasted } \\
\rho^{s}(t)\end{array}$ & $\begin{array}{l}\text { Forecasted } \\
D I(t)\end{array}$ & CCS & ECS & $P^{m s}(t)(\mathrm{MW})$ & $P^{c s}(t)(\mathrm{MW})$ & $P^{s b}(t)(\mathrm{MW})$ & $P^{s m}(t)(\mathrm{MW})$ & $R^{s}(t)(\mathrm{MW})$ \\
\hline $1: 00$ & 37.8 & 3 & 0 & 0 & 0 & 0 & 0 & 0 & 0 & 0 \\
\hline $2: 00$ & 37.1 & 3 & 0 & 0 & -1 & 10 & 0 & 0 & 0 & 10 \\
\hline $3: 00$ & 37.2 & 3 & 0 & 0 & -1 & 10 & 0 & 0 & 0 & 10 \\
\hline $4: 00$ & 37.5 & 3 & 0 & 0 & -1 & 1.7 & 0 & 0 & 0 & 1.7 \\
\hline $5: 00$ & 38.4 & 3 & 0 & 0 & 0 & 0 & 0 & 0 & 0 & 0 \\
\hline $6: 00$ & 40.8 & 8 & 0 & 0 & 1 & 0 & 0 & 7.5 & 0 & 2.5 \\
\hline $7: 00$ & 47.3 & 8 & 0 & 1 & -1 & 0 & 8.9 & 0 & 0 & 8.9 \\
\hline $8: 00$ & 53.7 & 8 & 0 & 1 & 0 & 0 & 0 & 0 & 0 & 10 \\
\hline 9:00 & 54.8 & 8 & 0 & 1 & 0 & 0 & 0 & 0 & 0 & 10 \\
\hline $10: 00$ & 54.5 & 10 & 0 & 1 & 0 & 0 & 0 & 0 & 0 & 10 \\
\hline $11: 00$ & 48.9 & 8 & 0 & 1 & 0 & 0 & 0 & 0 & 0 & 10 \\
\hline $12: 00$ & 45.6 & 8 & 0 & 1 & 0 & 0 & 0 & 0 & 0 & 10 \\
\hline $13: 00$ & 44.5 & 8 & 1 & 1 & 1 & 0 & 0 & 0 & 0 & 10 \\
\hline $14: 00$ & 42.5 & 8 & 0 & 1 & -1 & 0 & 10 & 0 & 0 & 10 \\
\hline $15: 00$ & 43.4 & 8 & 0 & 1 & -1 & 0 & 1.67 & 0 & 0 & 1.67 \\
\hline $16: 00$ & 48.4 & 8 & 0 & 1 & 1 & 0 & 0 & 0 & 0 & 10 \\
\hline $17: 00$ & 64.6 & 10 & 0 & 1 & 1 & 0 & 0 & 0 & 10 & 0 \\
\hline $18: 00$ & 60.0 & 10 & 0 & 1 & 0 & 0 & 0 & 0 & 8.3 & 0 \\
\hline $19: 00$ & 56.3 & 10 & 0 & 1 & 0 & 0 & 0 & 0 & 0 & 0 \\
\hline $20: 00$ & 50.2 & 8 & 0 & 1 & 0 & 0 & 0 & 0 & 0 & 0 \\
\hline $21: 00$ & 43.5 & 8 & 0 & 1 & 0 & 0 & 0 & 0 & 0 & 0 \\
\hline $22: 00$ & 41.8 & 8 & 0 & 1 & -1 & 0 & 10 & 0 & 0 & 10 \\
\hline 23:00 & 40.1 & 8 & 0 & 0 & 1 & 0 & 0 & 8.5 & 0 & 0 \\
\hline $24: 00$ & 37.8 & 3 & 0 & 0 & 0 & 0 & 0 & 0 & 0 & 0 \\
\hline
\end{tabular}

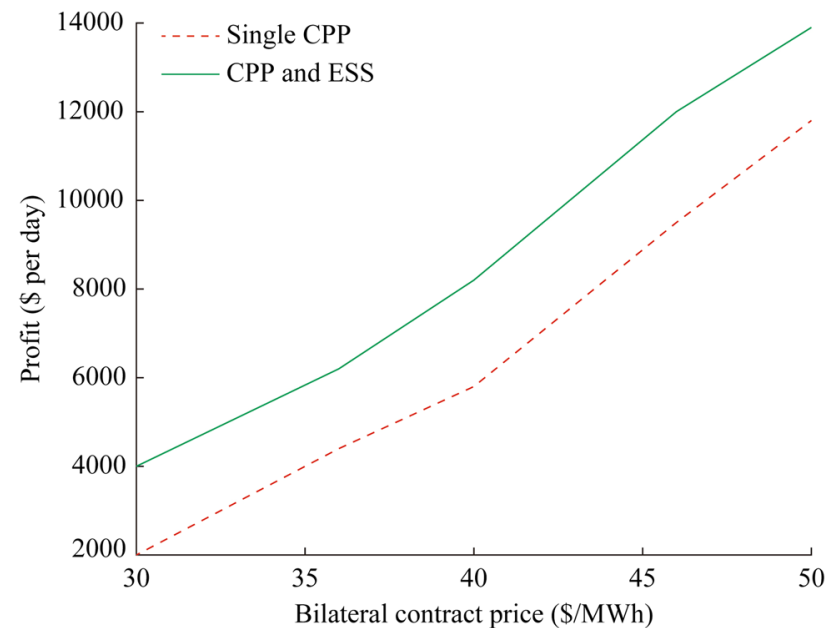

Fig. 3 GENCO's profit with respect to bilateral contract price for 15 MW bilateral contract (G1 + ESS)

changed in the presence of the ESS. In the absence of ESS, for prices lower than $45 \$ \mathrm{MWh}$ the CPP prefers to be dispatched for more hours than the combined CPP \& ESS.

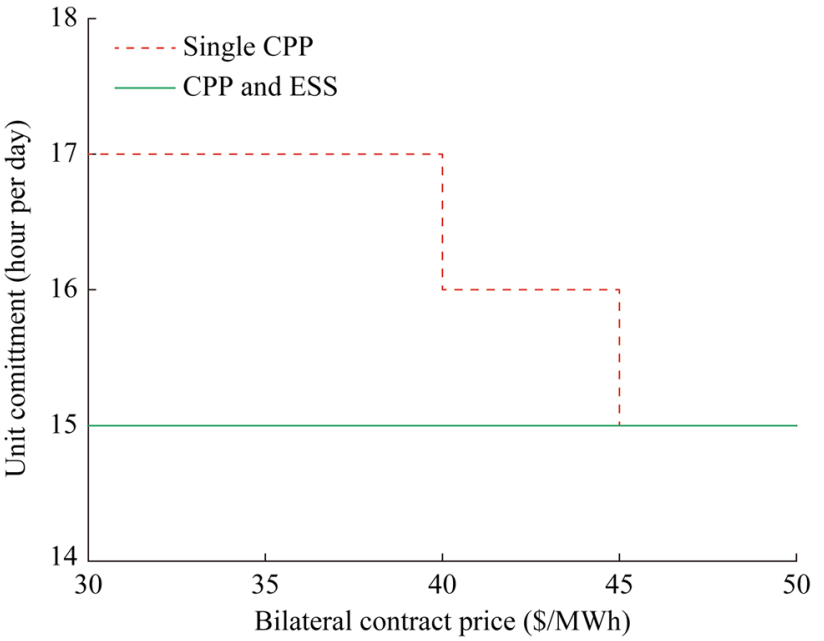

Fig. 4 Number of on-line hours of CPP with respect to bilateral contract price for $15 \mathrm{MW}$ bilateral contract (G1 + ESS)

In this situation, for combined ESS \& CPP, the CPP should not be $\mathrm{ON}$ to provide the bilateral contract. Indeed, for prices between 30 to $50 \$ / \mathrm{MWh}$, the GENCO prefers to use 


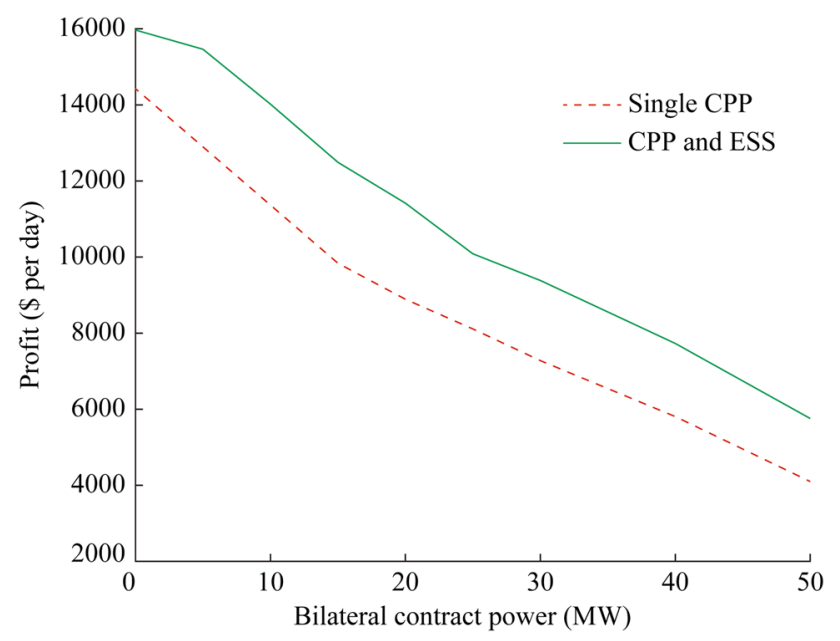

Fig. 5 GENCO's profit with respect to bilateral contract power with $45 \$ / \mathrm{MWh}$ price $(\mathrm{G} 1+\mathrm{ESS})$

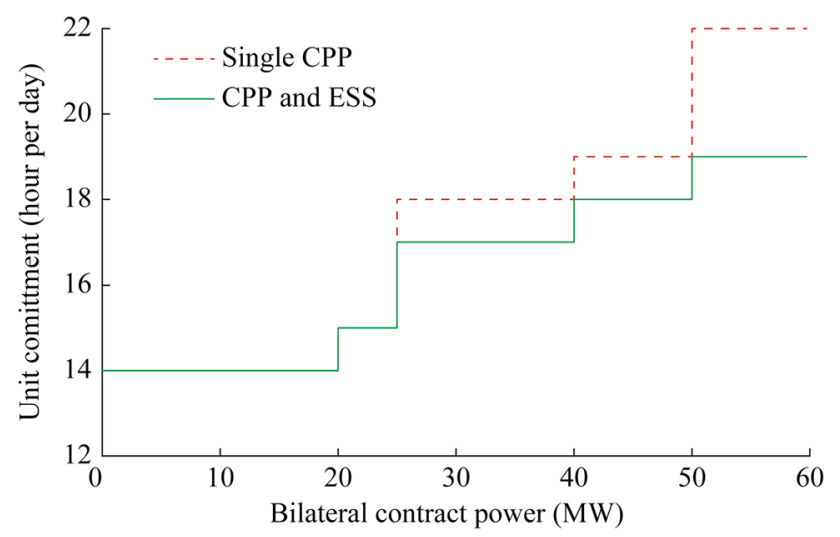

Fig. 6 On-line hours numbers of the CPP with respect to bilateral contract power with 45 \$MWh price (G1 + ESS)

the ESS or to buy energy from the market to provide the bilateral contract. For prices higher than $45 \$ / M W h$, despite the equal CPP commitments, the profit of the GENCO for the combined CPP and ESS is higher than that of the single CPP, as shown in Fig. 3.

For the $45 \$ / \mathrm{MWh}$ bilateral contract price, the GENCO's profit, with and without the ESS, with respect to the bilateral contract power is depicted in Fig. 5. The results show that the GENCO's profit in the presence of the ESS is higher than that of the single CPP for all bilateral contract powers. The number of CPP commitment hours in this condition, with and without the ESS, is displayed in Fig. 6. In this condition, the number of CPP commitment hours in the presence of the ESS is equal to or lower than that without ESS.

According to the study's results, the advantages of using the ESS beside the CPP are as follows:
Table 3 Coordinated PBUC and PBSC profit with respect to power and energy capacities of the ESS (G1 + ESS) \$/MWh

\begin{tabular}{lllll}
\hline $\begin{array}{l}\text { Energy capacity } \\
\text { (MWh) }\end{array}$ & \multicolumn{5}{l}{ Power capacity (MW) } \\
\cline { 2 - 5 } & 5 & 10 & 15 & 20 \\
\hline 5 & 13070.03 & 13867.52 & 14362.76 & 15026.15 \\
10 & 13253.90 & 14002.85 & 14605.55 & 15238.94 \\
20 & 13711.81 & 14270.58 & 14999.27 & 15660.86 \\
40 & 13976.93 & 14406.75 & 15275.56 & 15901.95 \\
\hline
\end{tabular}

1) GENCO's profitability increase: in the presence of the ESS, because of its technical properties, the profitability opportunities such as price arbitrage are added.

2) Reduction in the number of on-line hours of the CPP: as given in the results, in the presence of the ESS, the number of CPP commitment hours sometimes reduces by 10 percent. This is effective in the operation improvement and lifetime increment of the CPP.

3) Operation improvement of the CPP: the participation of the CPP in the spinning reserve market needs a rapid response and leads to cycling problems. The ESS can help the GENCO to participate in the spinning reserve market and reduce the CPP usage in this market. This decreases the CPP costs and increases the CPP lifetime. Besides, the ESS can help the CPP to operate near its nominal capacity. Without the ESS, at some hours the CPP may have to be committed and generate lower than its rated power for supply according to its bilateral contract $(6: 00,7: 00,22: 00$ and 23:00 in Table 2). In such a condition, with the presence of the ESS, the CPP can remain OFF and the ESS can be discharged to supply the bilateral contract (6:00 and 23:00) or the CPP can be ON and provide the bilateral contract and also charge the ESS (7:00 and 22:00). Therefore the CPP could operate near to its rated power.

The profit of the coordinated PBUC and PBSC is represented in Table 3, for a 15 MW @ 50 \$/MWh (i.e. 15 MW generation at $50 \$ / \mathrm{MWh}$ ) bilateral contract and for different ESS power and energy capacities. Power capacity is the maximum instantaneous output that the ESS can provide, and energy capacity is the amount of electrical energy that the ESS can store. As shown in this table, the profit of the GENCO has increased as the ESS power and energy capacities enhance. This is due to the more energy storing capability and instantaneous output of the ESS and then more profitability opportunities. The result of employing more than one ESS is implicitly represented in Table 3. If two similar ESSs (10 MW/20 MWh) are installed beside the G1, we deal with $20 \mathrm{MW} / 40 \mathrm{MWh}$ 


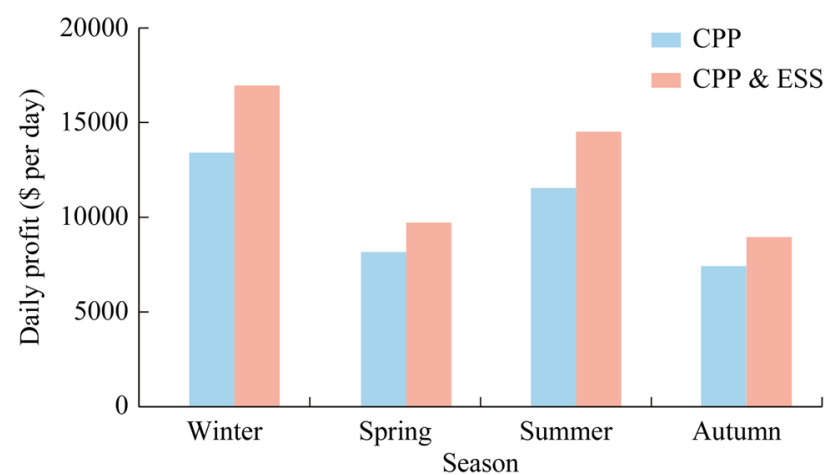

Fig. 7 Daily profits of GENCO with and without ESS for four sample days $(\mathrm{G} 1+\mathrm{ESS})$

storage capacities. This table shows that the profitability of two ESSs is more than one ESS. But there is the question of whether this surplus profit encourages the GENCO to invest in more than one ESS. This question will be answered in the next studies of the authors.

The daily profit of G1, with and without the ESS (10 $\mathrm{MW} / 20 \mathrm{MWh}$ ), and for four sample days in different seasons in 2015 with a 15 MW@ 50 \$/MWh bilateral contract, is depicted in Fig. 7. The results show that in the presence of the ESS, the GENCO's profitability in the winter and summer are relatively higher than that in the spring and autumn. The reason is that the differences between the hourly energy and ancillary service prices in the winter and summer are usually higher than those in the spring and autumn. Hence, in the winter and summer, the ESS can earn higher profit by price arbitraging.

The energy and spinning reserve prices are the main inputs of the proposed model. Thus, the model output is certainly different for the different markets with different prices. But, one of the main properties of the proposed model is that the arbitraging option has not only been provided by the energy market. The ESS can be charged using the adjacent CPP in the GENCO and receiving power from the energy market, and can be discharged for the bilateral contract and the energy and spinning reserve markets. Therefore, the ESS provides the arbitraging option between the internal generation of the GENCO, bilateral contract, and the energy and spinning reserve

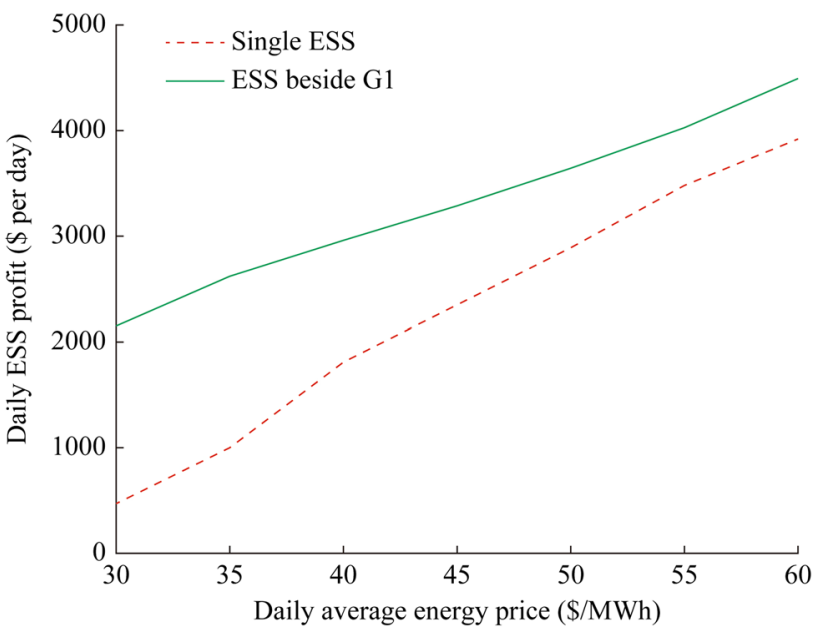

Fig. 8 Daily ESS profit with respect to daily average energy price (single ESS and combination of ESS \& CPP)

markets. Besides, the ESS improves the technical performance of the ESS and decreases the CPP operation costs. Thus, the presence of the ESS does not only depend on the market prices. The sensitivity on the generation unit's prices is also reduced.

The impacts of installing the ESS beside the CPP for G1, G2 and G3 are presented in Table 4. It is supposed that the GENCO has a $15 \mathrm{MW} @ 40$ \$/MWh bilateral contract. At first, the profits of the ESS for sample days in different seasons are represented in this table. The daily profit of employing ESS is equal to the daily profit of the GENCO in the presence of the ESS minus the daily profit of the GENCO without the ESS.

The results show that the daily profit of the GENCO increases in the presence of the ESS. This increase in profit for the base load power plant is more than other CPPs because G2 has more price arbitrage opportunities. Also, the ESS profits in the winter and summer are relatively higher than in the spring and autumn. The decrements of the on-line hours of the CPP are then presented in Table 4. The ESS decreases more generation commitment in G3 than in other CPPs. In this situation the ESS decreases the hours when the CPP must be ON and generates power lower than rated.

Table 4 Results of studies performed on G1, G2 and G3

\begin{tabular}{|c|c|c|c|c|c|c|c|c|c|c|c|c|}
\hline \multirow[t]{2}{*}{ CPP } & \multicolumn{4}{|c|}{ Daily profit of ESS (\$/day) } & \multicolumn{4}{|c|}{ Daily on-line hours reduction (hour/day) } & \multicolumn{4}{|c|}{ Daily cycling capability (MW/(hour·day)) } \\
\hline & Winter & Spring & Summer & Autumn & Winter & Spring & Summer & Autumn & Winter & Spring & Summer & Autumn \\
\hline G1 & 3287 & 2657 & 3196 & 2603 & 1 & 2 & 1 & 2 & 38.1 & 31.4 & 36.8 & 32.7 \\
\hline G2 & 3553 & 2910 & 3365 & 2830 & 0 & 0 & 0 & 0 & 42.3 & 35.1 & 41.2 & 34.9 \\
\hline G3 & 2858 & 3305 & 2721 & 2295 & 1 & 3 & 1 & 3 & 31.7 & 27.4 & 31.4 & 27.6 \\
\hline
\end{tabular}


At last, the increases of cycling capability of the GENCO in the presence of the ESS are shown. Cycling capacity is defined as the output power change during one hour $(\Delta P /$ hour). The results show that the cycling capability of the GENCO increases in the presence of the ESS. The ESS increases the cycling capability of G2 more than other CPPs.

For more assessment of the price changes impacts on the results a sensitivity analysis is carried out. In Fig. 8, the daily ESS profit with respect to the daily average energy price is depicted for two cases (single ESS and combination of ESS \& CPP). As the average energy price increases, the ESS profit increases in both cases. But with decreasing the average energy price, the rate of ESS profit reduction for the combination of ESS \& CPP is lower than the single ESS because there is only energy exchange opportunity between the ESS and the market for single ESS. Thus the ESS profit depends only on the energy price. But if the ESS is coordinated with the CPP, considering the energy exchange opportunities between the ESS, CPP and the market and also the impacts of the ESS in the performance of the CPP, the price reduction has a lower impact on the ESS profitability. But if the ESS is connected to the CPP, considering the energy exchange opportunities between the ESS, CPP and the market and also the impacts of the ESS in the performance of the CPP, the price reduction has lower impact on the ESS profitability.

The presentation of the model proposed for the coordinated PBUC and PBSC opens an important question. The question is that which ESS technologies are suitable to be installed beside the CPPs.

The main factors for selection of ESS technologies are the capital cost, lifetime, cycling capacity, and round trip efficiency. The capital cost includes two costs related to the power capacity and energy capacity of the ESS. Cycling capacity of the ESS is the maximum possible number of charging/discharging cycles of the ESS (one cycle includes one charging and one discharging) in its lifetime. The following assumptions are considered in the economic assessment:
1) The ESS has almost two full charging/discharging cycles in a day.

2) The stored energy in the ESS in each day should be released at the same day.

3) Four sample days in different seasons are studied to determine the yearly profit.

4) The average energy price in each season is considered as the bilateral contract price of that season.

5) A bilateral contract power of $15 \mathrm{MW}$ is considered for using a $10 \mathrm{MW} / 20 \mathrm{MWh}$ ESS beside the CPP.

6) Annual interest rate is considered to be $8 \%$.

7) It is supposed that the GENCO's bids are accepted in the energy and spinning reserve markets.

The characteristics of a number of ESS technologies for economic assessment are presented in Table $5[29,30]$. Compressed air energy storage (CAES), lead acid (LA) battery, sodium sulfur ( $\mathrm{NaS})$ battery, vanadium redox battery (VRB), lithium ion (Li-ion) battery are studied in this paper. CAES is a common storage technology which is used for bulk storing [31]. Nowadays, large-scale battery energy storage systems are used around the world $[5,32,33]$. In this paper, for the economic assessment, the payback period of investment in the ESS is evaluated. Cycling capacity is a serious limitation of the ESS.

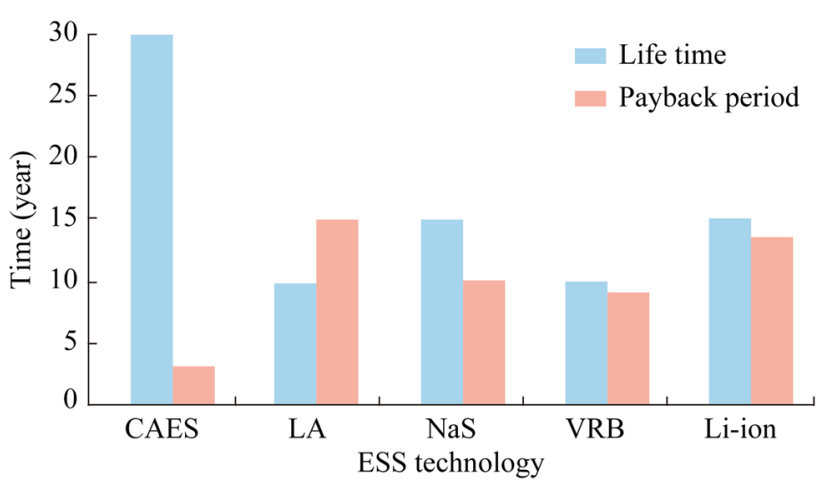

Fig. 9 Comparison of life time and payback period of different ESS technologies (G1 + ESS)

Table 5 Characteristics parameters of ESS technologies

\begin{tabular}{llllll}
\hline ESS technologies & \multicolumn{2}{l}{ Capital cost } & Cycling capacity & Life time (year) & Round trip efficiency $(\%)$ \\
\cline { 2 - 5 } & Energy cost $(\$ / \mathrm{kWh})$ & Power cost $(\$ / \mathrm{kW})$ & & \\
\hline CAES & 10 & 200 & 30000 & 30 & 80 \\
LA & 300 & 400 & 1000 & 10 & 85 \\
$\mathrm{NaS}$ & 250 & 150 & 2500 & 15 & 85 \\
VRB & 200 & 300 & 12000 & 10 & 80 \\
Li-ion & 500 & 200 & 10000 & 15 & 85 \\
\hline
\end{tabular}




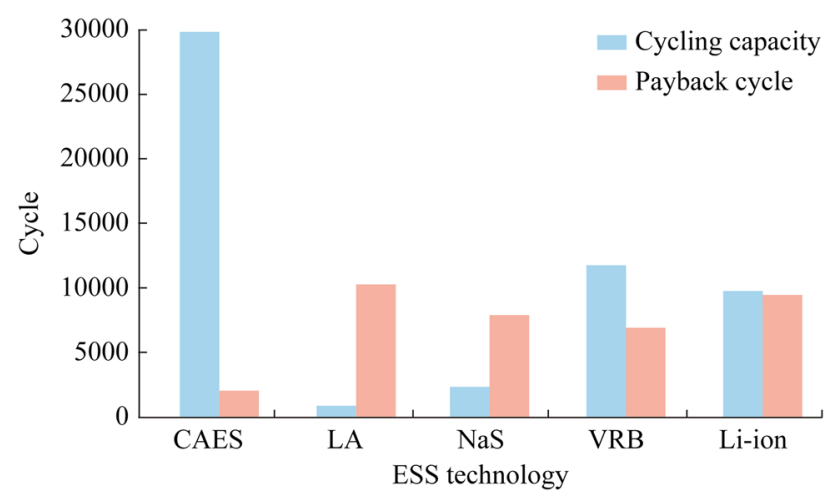

Fig. 10 Comparison of cycling capacity and number of payback cycles for different ESS technologies (G1 + ESS)

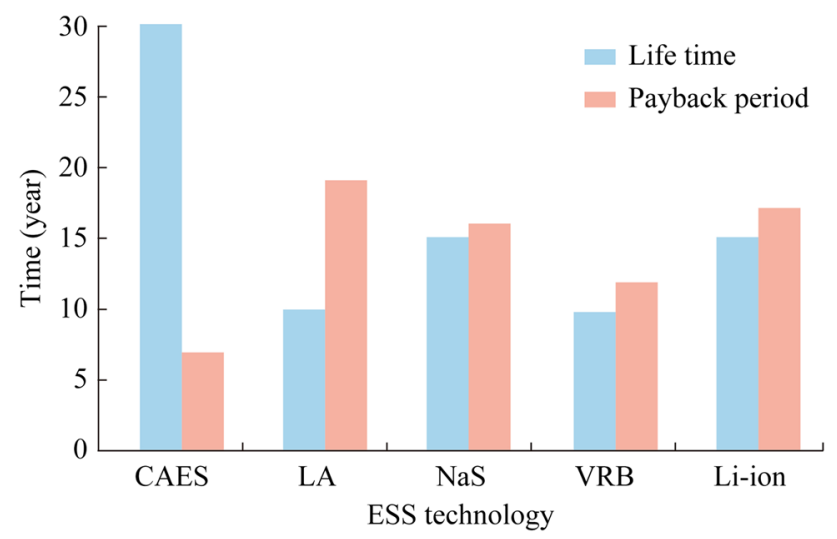

Fig. 11 Comparison of life time and payback period for different single ESS technologies

Therefore, the number of payback cycles is proposed as an economic criterion in this paper. The number of payback cycles is defined as the number of charging/discharging cycles required to recover the capital cost of the ESS.

The results of economic assessment to determine the potential of ESSs to install beside the CPPs are displayed in Figs. 9 and 10. In Fig. 9, the lifetime and payback period of the ESS technologies are presented. This figure shows that the payback periods of the CAES, NaS, VRB, and Liion are lower than their lifetimes. As shown in this figure, the LA is not a suitable choice for investment. In Fig. 10, the number of payback cycles for the five ESS technologies are compared with their cycling capacity. As shown in Fig. 10, the number of payback cycles for the CAES, VRB, and $\mathrm{Li}$-ion are lower than their cycling capacity. Figure 10 shows that the NaS and LA are not suitable for installing beside the CPP, because the number of cycles required to recover the capital cost of these technologies are higher than their cycling capacities. Considering the results in the Figs. 9 and 10, we conclude that the CAES, VRB and Liion are the economic choices from the GENCO's viewpoint. The NaS, as well, could be economic if other profitability opportunities and long-term storing chance (e.g., weekly storing) are considered.

In Fig. 11, the lifetime and payback period of the single ESS technologies are compared. Here it is supposed that the ESS technologies have been used individually and have not been installed beside the other power plants. The ESS can only exchange power with the market. The results show that the most of ESS technologies are not economic and only CAES is suitable for investing individually.

The future work of the authors includes the following issues:

1) Determining the optimal capacity of the candidate ESS technologies to use beside the CPPs.

2) Studying the impact of the ESSs on the different CPP technologies.

\section{Conclusion}

In this paper a new model is presented to coordinate the PBUC and PBSC, from a price taker GENCO's point of view. This model determines the status of a CPP, and charging, discharging and idle modes of an ESS to participate in the day-ahead energy and spinning reserve markets and bilateral contract provision. Results show that in the presence of the ESS, the operation of the CPP is improved, in addition of the increase of GENCO's profitability. Combining the ESS and the CPP reduces the number of on-line hours of the CPP. The economic assessment concludes that the CAES, Li-ion, and VRB are appropriate technologies to be used beside the CPP. Also, the economic results show that only CEAS is suitable for individual investment. But using the proposed model in this paper, some other ESS technologies are suitable for the individual.

Open Access This article is distributed under the terms of the Creative Commons Attribution 4.0 International License (http:// creativecommons.org/licenses/by/4.0/), which permits unrestricted use, distribution, and reproduction in any medium, provided you give appropriate credit to the original author(s) and the source, provide a link to the Creative Commons license, and indicate if changes were made.

\section{References}

[1] Yao L, Yang B, Cui H et al (2016) Challenges and progresses of energy storage technology and its application in power systems. J Mod Power Syst Clean Energy 4(4):519-528

[2] Li X, Yao L, Hui D (2016) Optimal control and management of a large-scale battery energy storage system to mitigate fluctuation and intermittence of renewable generations. J Mod Power Syst Clean Energy 4(4):593-603 
[3] Chen J, Song X (2015) Economics of energy storage technology in active distribution networks. J Mod Power Syst Clean Energy 3(4):583-588

[4] Sangster JA (2016) Massive energy storage systems enable secure electricity supply from renewables. J Mod Power Syst Clean Energy 4(4):659-667

[5] Liu WX, Niu SY, Xu HT (2017) Optimal planning of battery energy storage considering reliability benefit and operation strategy in active distribution system. J Mod Power Syst Clean Energy 5(2):177-186

[6] Sheibani MR, Yousefi GR, Latify MA et al (2018) Energy storage system expansion planning in power systems: a review. IET Renew Power Gen 12(11):1203-1221

[7] Van den Bergh K, Delarue E (2015) Cycling of conventional power plants: technical limits and actual costs. Energy Convers Manage 97:70-77

[8] Korpas K, Holen AT (2006) Operation planning of hydrogen storage connected to wind power operating in a power market. IEEE Trans Energy Convers 21(3):742-749

[9] Khodayar M, Shahidehpour M (2013) Stochastic price-based coordination of intrahour wind energy and storage in a generation company. IEEE Trans Sustain Energy 4(3):554-562

[10] Gomes I, Pousinho H, Melicio R et al (2017) Stochastic coordination of joint wind and photovoltaic systems with energy storage in day-ahead market. Energy 124:310-320

[11] Ding H, Pinson P, Hu Z et al (2017) Optimal offering and operating strategy for a large wind-storage system as a price maker. IEEE Trans Power Syst 32(6):4904-4913

[12] Pandzic H, Kuzle I, Capuder T (2013) Virtual power plant midterm dispatch optimization. Appl Energy 101:134-141

[13] Pandzic H, Morales JM, Conejo AJ et al (2013) Offering model for a virtual power plant based on stochastic programming. Appl Energy 105:282-292

[14] Peik-Herfeh M, Seifi H, Sheikh-El-Eslami MK (2013) Decision making of a virtual power plant under uncertainties for bidding in a day-ahead market using point estimate method. Int J Electr Power Energy Syst 44:88-98

[15] Ghahgharaee Zamani A, Zakariazadeh A, Jadid S (2016) Dayahead resource scheduling of a renewable energy based virtual power plant. Appl Energy 169:282-292

[16] Ghahgharaee Zamani A, Zakariazadeh A, Jadid S et al (2016) Stochastic operational scheduling of distributed energy resources in a large scale virtual power plant. Int J Electr Power Energy Syst 82:608-620

[17] Shayegan-Rad A, Badri A, Zanganeh A (2017) Day-ahead scheduling of virtual power plant in joint energy and regulation reserve markets under uncertainties. Energy 121:114-125

[18] Smith AD, Mago PJ, Fumo N (2013) Benefits of thermal energy storage option combined with CHP system for different commercial building types. Sustain Energy Technol Assess 1:3-12

[19] Katulic S, Cehil M, Bogdan Z (2014) A novel method for finding the optimal heat storage tank capacity for a cogeneration power plant. Appl Therm Eng 65(1-2):530-538

[20] Sartor K, Dewallef P (2018) Integration of heat storage system into district heating networks fed by a biomass CHP plant. J Energy Stor 15:350-358

[21] Kia M, Nazar MS, Sepasian MS et al (2017) Optimal day ahead scheduling of combined heat and power units with electrical and thermal storage considering security constraint of power system. Energy 120:241-252

[22] Nuytten T, Claessens B, Paredis K et al (2013) Flexibility of a combined heat and power system with thermal energy storage for district heating. Appl Energy 104:583-591

[23] Chen X, Kang C, O'Malley M et al (2015) Increasing the flexibility of combined heat and power for wind power integration in China: modeling and implications. IEEE Trans Power Syst 30(4):1848-1857

[24] Kubic ML, Coker PJ, Hunt C et al (2012) The role of conventional generation in managing variability. Energy Policy 50:253-261

[25] Hosseini-Firouz M (2013) Optimal offering strategy considering the risk management for wind power producers in electricity market. Int J Electr Power Energy Syst 49:359-368

[26] Tan Z, Ju L, Li H et al (2014) A two-stage scheduling optimization model and solution algorithm for wind power and energy storage system considering uncertainty and demand response. Int J Electr Power Energy Syst 63:1057-1069

[27] Shahidehpour M, Yamin H, Li Z (2002) Market operations in electric power systems, forecasting, scheduling and risk assessment, 1st edn. Wiley, New York

[28] NYISO (2015b) NYISO energy prices data. Available: http:// www.nyiso.com/public/markets_operations/market_data/pricing_ data/index.jsp. Accessed Oct 2016

[29] Luo X, Wang J, Dooner M et al (2015) Overview of current development in electrical energy storage technologies and the application potential in power system operation. Appl Energy 137:511-536

[30] Akinyele D, Rayudu R (2014) Review of energy storage technologies for sustainable power networks. Sustain Energy Technol Assess 8:74-91

[31] Chen L, Zheng T, Mei S et al (2016) Review and prospect of compressed air energy storage system. J Mod Power Syst Clean Energy 4(4):529-541

[32] Poullikkas A (2013) A comparative overview of large-scale battery systems for electricity storage. Renew Sust Energy Rev 27:778-788

[33] Yang XF, Xue Y, Chen BW et al (2017) Reverse-blocking modular multilevel converter for battery energy storage systems. J Mod Power Syst Clean Energy 5(4):652-662

Mohammad Reza SHEIBANI received the B.Sc. degree in electronic engineering from the Isfahan University of Technology, Isfahan, Iran, in 2008, and the M.Sc. degree from the University of Kashan, Kashan, Iran, in 2011. Currently, he is Ph.D. candidate in the Isfahan University of Technology, Isfahan, Iran. His research interests are energy storage systems, power system planning and operation, and electricity market.

Gholam Reza YOUSEFI received his B.Sc. degree in Electrical Engineering from Isfahan University of Technology, Isfahan, Iran, in 1994, and his M.Sc. and Ph.D. degrees in Electrical Engineering from Tarbiat Modares University, Tehran, Iran, in 1997 and 2001, respectively. He had been with Midwest Service Center, Hobart, IN, USA, and Pacific Gas and Electric Company (PG\&E), San Francisco, USA. Currently, he is with the Department of Electrical and Computer Engineering, Isfahan University of Technology, as an Associate Professor. His research interests are power systems operation and planning, as well as electricity markets and smart grids.

Mohammad Amin LATIFY received the B.Sc. degree from Isfahan University of Technology, Isfahan, Iran, in 2002 in electrical engineering. He also received the M.Sc. degree from Ferdowsi University of Mashhad, Mashhad, Iran, in 2005, and the Ph.D. degree from Tarbiat Modares University, Tehran, Iran, in 2012, in electrical engineering with honors. He is currently an Assistant Professor at Isfahan University of Technology, Isfahan, Iran. His research interests are electricity markets and economics, power system operation and planning and optimization theory applications. 